\title{
Text-Based Intelligent Learning Emotion System
}

\author{
Mohammed Abdel Razek ${ }^{1,2}$, Claude Frasson ${ }^{3}$ \\ ${ }^{1}$ Research and Development Department, Deanship of Distance Learning, King Abdulaziz University, Jeddah, Kingdom of Saudi \\ Arabia \\ ${ }^{2}$ Math \& Computer Science Department, Faculty of Science, Azhar University, Cairo, Egypt \\ ${ }^{3}$ Département d'informatique \& de recherche opérationnelle Université de Montréal, Québec, Canada \\ Email:maabdulraze1@kau.edu.sa,frasson@iro.umontreal.ca
}

How to cite this paper: Razek, M.A. and Frasson, C. (2017) Text-Based Intelligent Learning Emotion System. Journal of Intelligent Learning Systems and Applications, 9, 17-26.

https://doi.org/10.4236/jilsa.2017.91002

Received: September 1, 2016

Accepted: February 3, 2017

Published: February 6, 2017

Copyright $\odot 2017$ by authors and Scientific Research Publishing Inc. This work is licensed under the Creative Commons Attribution International License (CC BY 4.0).

http://creativecommons.org/licenses/by/4.0/

\begin{abstract}
Nowadays, millions of users use many social media systems every day. These services produce massive messages, which play a vital role in the social networking paradigm. As we see, an intelligent learning emotion system is desperately needed for detecting emotion among these messages. This system could be suitable in understanding users' feelings towards particular discussion. This paper proposes a text-based emotion recognition approach that uses personal text data to recognize user's current emotion. The proposed approach applies Dominant Meaning Technique to recognize user's emotion. The paper reports promising experiential results on the tested dataset based on the proposed algorithm.
\end{abstract}

\section{Keywords}

Text Based Emotion, Intelligent Learning System, Dominant Meaning

\section{Introduction}

In collaborative chatting between users, emotions are an important aspect. The detection of the exchange of emotions among users through text messages can help for delivering right emotion in the right time. Several researches used textbased emotion to predict and classify the emotion types, such as [1] [2] [3] and [4]. Jraidi et al. [5] show the impact of using emotion in intelligent system and show how these emotions oriented toward developing emotionally sensitive tutors.

This paper presents a new technique based on Dominant Meaning Technique [6] and Appraisal Method [7] to classify a text to a suitable emotion. The domi- 
nant meanings definition is known as "the set of keywords that best fit an intended meaning of a target word" [6]. This technique sees the target meaning as a master word. Some slave words are to be added to the master to clarify the target meaning. For example, a word bank has several meaning, 1) a financial bank with slaves' words such as statement, loan, and rate, 2) blood bank with slaves such as medical, transfusion, and human body, 3) river bank with slaves such as land, lake, and edge.

Appraisal is a linguistic theory that tries to model language's capability to definite opinions and attitudes within text [7]. The appraisal method contains three distinct aspects: Attitude, Engagement, and Graduation. In this paper, we adopt attitudes in its classification. Attitudes are separated into three categories: Affect, Judgment, and Appreciation. Attitude is defined as a mode that anyone acts in a specific condition and shows how he feels [8]. These aspects embody the capability to express emotional, moral, and aesthetic feelings respectively [9]. For example, "when I was in grade 11 in the school, I was punished for no serious mistake of mine" another sentence "when I was in grade 11 in the school, I got an award for my excellence". Using the dominant meaning methods, the words "punish, and mistake" lead the first sentences to a negative emotion, however, the words "award and excellence" classify the second sentences to a positive emotion.

Detecting emotion from text is useful in understanding users' feelings towards particular discussion in intelligent learning system. To test our algorithm, we use ISEAR (International Survey on Emotion Antecedents and Reactions), dataset collected by Klaus R. Scherer and Harald Wallbott [9]. ISEAR dataset contains seven major emotions: joy, fear, anger, sadness, disgust, shame, and guilt. The process to classify sentences in this work involves two main steps: representing $40 \%$ of dataset to allow learning, extract features based on appraisal method, create dominant meaning hierarchy, train a classifier on prepared examples, and then using the classifier to predict a category.

The remainder of this paper is organized as follows. Section 2 presents the methodology to detect the emotion and how to construct dominant meaning tree. Section 3 describes experiments and discusses the results. Finally, Section 4 summarizes the conclusion.

\section{Emotion Detection Methodology}

The architecture of the proposed system contains two stages: training stage, and classification stage. The training stage happens on the server side. We apply the dominant meaning methods [6] on the ISEAR dataset [9] to form the hierarchy tree. Based on the ISEAR, the tree consists of seven concepts: joy, fear, anger, sadness, disgust, shame, and guilt.

The classifier unit receives two types of information. A hierarchy tree for dominant meaning for seven classes and ISEAR examples. The classifier in general uses a large amount of labeled training data for text classification, which is a labor-intensive and time-consuming task. In contrast, our approach is to con- 
struct the dominant meaning tree and then use this tree to classify incoming examples from Emotion Models unit. This unit contains two types of set of words. First, set coming from Emotion Agent, which extract some features from Chatting GUI unit during the chatting between users, remove stop words, and reformulate in the way Emotion unit can deal with it. Stop words are those that occur commonly but are too general-such as "the", "an", "a", "to", etc. The algorithm removed the stop words from the collection. Emotion agent use Emotion Algorithm to assign an emotion for each set of features based on the emotion models coming from emotion models unit. After determining the emotion, Emotion Expression assigns a suitable expression for it and sends it to be shown in the Chatting GUI (see Figure 1).

\subsection{Constructing Emotion Dominant Meaning Tree}

To represent the proposed approach to classify sentiment, suppose that the collection consists of $m$ emotion, i.e. $E=\left\{\zeta_{k}\right\}_{i=1}^{m}$. Given the limited set of examples for each emotion, we try to represent the collection as a hierarchy of dominant meanings.

In this definition, each emotion is represented by a finite set of examples $\zeta_{k}=\left\{D_{v}^{k} \mid v=1, \cdots, r_{k}\right\}$. The question now is how can we use those examples to construct dominant meanings of the corresponding emotion? In other words, those examples include some words that almost come with the corresponding emotion. The challenge is how to determine those words.

Each example is represented by a fixed set of words $D_{v}^{k}=\left\{w_{j v}^{k} \mid j=1, \cdots, n_{v}\right\}$.

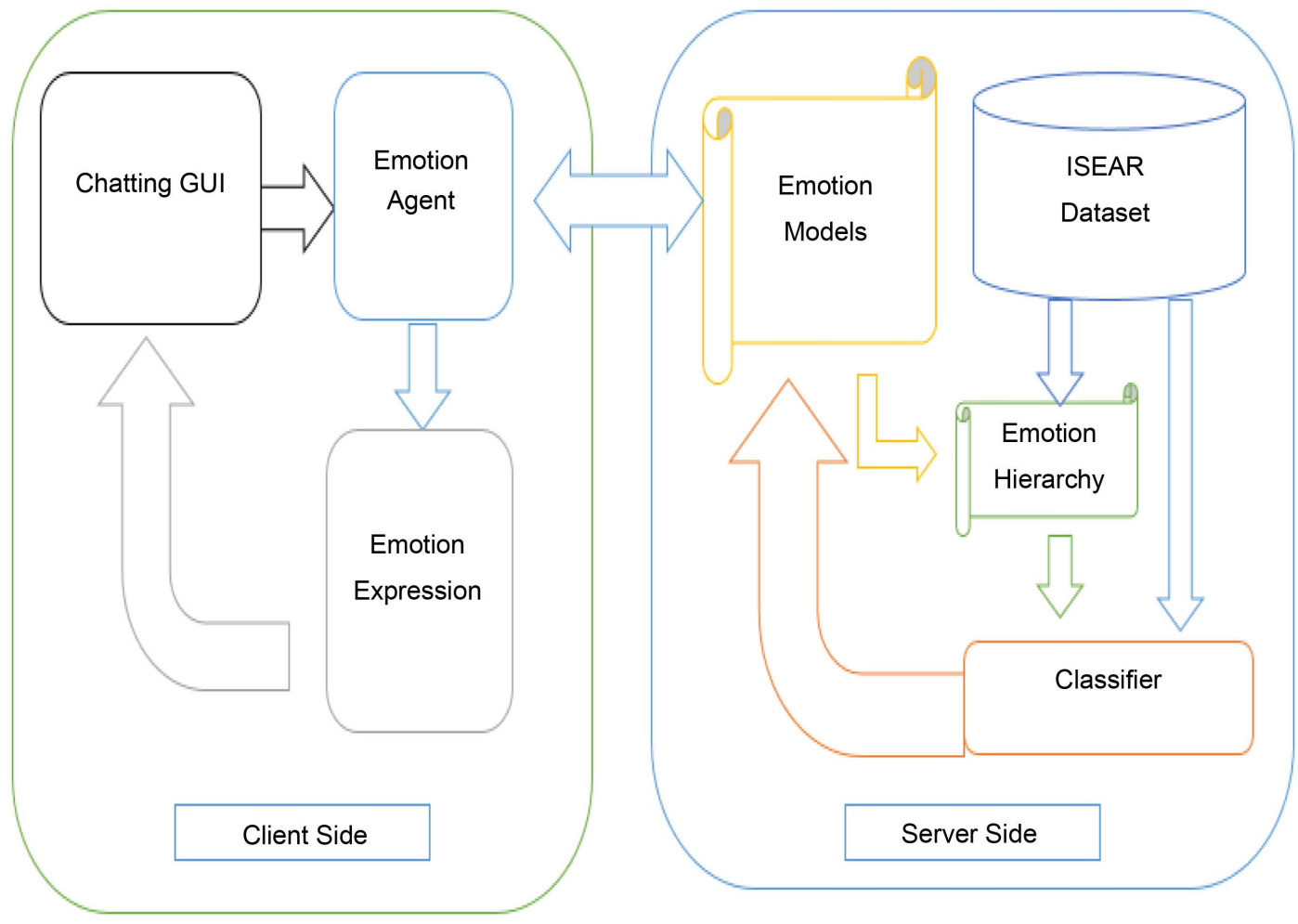

Figure 1. Architecture of the emotion detection system. 
The $w_{j v}^{k}$ 's represent the frequency of word $w_{j}$ occurs in example $D_{v}^{k}$ which belongs to emotion $\zeta_{k}$. This frequency is computed as the number of times that the $w_{j}$ occurs in the $D_{v}^{k}$.

Our goal is to choose the top- $T$ words, which can represent the dominant meanings of emotion $\zeta_{k}$. To do that, we proceed as follows. Suppose that word $w_{c}^{k}$ symbolizes emotion $\zeta_{k}$.

- Calculate the values of

$$
w_{j v}^{k} \forall j, v .
$$

- Suppose that $\beta_{k v}$ is the frequency of emotion $\zeta_{k}$, which appears in example $D_{v}^{k}$, where $v=1, \cdots, r_{k}$.

- Calculate the maximum value of $\beta_{k v} \forall v$,

$$
F_{c}^{k}=\operatorname{Max}_{v=1, \cdots, r_{k}}\left\{\beta_{k v}\right\} .
$$

- Calculate the maximum value of $w_{j v}^{k} \forall j, v$,

$$
F_{w_{j}}^{k}=\underset{v=1, \cdots, r_{k}}{\operatorname{Max}}\left\{w_{j v}^{k}\right\}
$$

where $v=1, \cdots, r_{k}$

- Choose $F_{c}^{k}$, which satisfies $0 \leq F_{w_{j}}^{k} \leq F_{c}^{k}$.

- Finally, consider the dominant meaning probability

- $P_{k j}=P_{k j}\left(w_{j} \mid \zeta_{k}\right)=\frac{1}{r_{k}}\left[\sum_{v=1}^{r_{k}} \frac{w_{j v}^{k}}{F_{c}^{k}}\right]$.

$j=1, \cdots, n_{v}, k=1, \cdots, m$

Therefore, we divide $w_{j v}^{k}$ by the maximum value $F_{c}^{k}$ of the frequency of $\zeta_{k}$, and then we normalize the results by dividing by the number of examples $r_{k}$ in collection $\zeta_{k}$. Based on formula (3), we clearly have $0 \leq P_{k j}\left(w_{j} \mid \zeta_{k}\right) \leq 1$.

\subsection{Constructing Emotion Dominant Meaning Models}

The proposed system creates sevens models one for each emotion: joy, fear, anger, sadness, disgust, shame, and guilt.

For each emotion $\zeta_{k}$, we have a collection of $N$ examples $\zeta_{k}=\left\{D_{v}^{k} \mid v=1, \cdots, r_{N}\right\}$. For each collection, we apply the formula from (1) to (4).

After applying formulas, we get a set of dominant meanings each word in the set has $P_{k i}$ value for a word $w_{i}$ and in emotion $\zeta_{k}$.

We rank the terms of collection $\left\{P_{k 1}, P_{k 2}, \cdots, P_{k n}\right\}$ in decreasing order according to formula (4). As a result, the dominant meanings of the emotion $\zeta_{k}$ can be represented by the set of words that is corresponds to the set $\left\{P_{k 1}, P_{k 2}, \cdots, P_{k n}\right\}$; i.e. $\zeta_{k}^{n}=\left\{w_{k 1}, w_{k 2}, \ldots, w_{k n}\right\}$.

Therefore, we select the top- $N$ values of $P_{k j}$ to form motion dominant meaning tree (EDMT). EDMT represents seven emotions suggested by (Klaus, 1994) as a tree. Each emotion is joined with a slave word. This slave is represent a dominant meaning and associated with the dominant meaning probability of that emotion as shown in Figure 2. In this paper we put the top- $N$ of $P_{k j}$ values as an arbitrary value. 


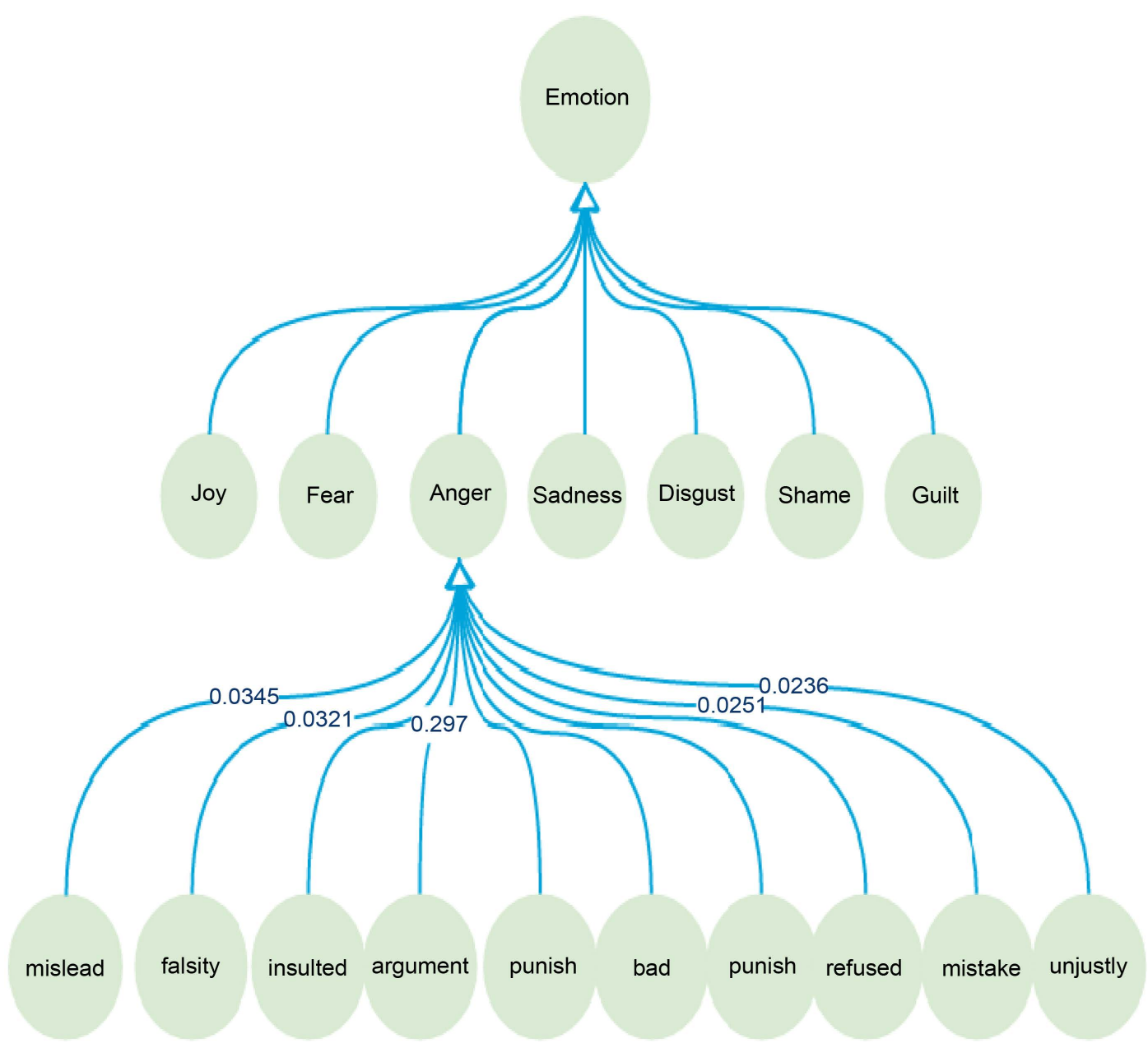

Figure 2. Emotion dominant meaning tree.

Accordingly, we can create seven models to represent the emotion. Each model is a set called emotion dominant meaning models $\left(\Gamma_{k}\right)$. Each $\Gamma_{k}$ contains the top- $N$ dominant meaning probability:

$$
\Gamma_{k}=\left\{P_{k 1}, P_{k 2}, \cdots, P_{k 10}\right\} .
$$

The corresponding word set of $\Gamma_{k}$ is represented as: $\zeta_{k}^{N}=\left\{w_{k 1}, w_{k 2}, \cdots, w_{k 10}\right\}$. For a new example $\psi=\left\{e_{1}, e_{2}, \cdots, e_{l}\right\}, e_{l}$ represents a word in the new example. For each emotion, we compute the model value $\Omega_{k}$ as flowing:

$$
\Omega_{k}(\psi)=\frac{1}{N} \sum_{i=1}^{N} \alpha_{i} P_{k i}
$$

where

$$
\alpha_{i}=\left\{\begin{array}{ll}
1 & e_{i} \in \zeta_{k}^{N} \\
0 & e_{i} \notin \zeta_{k}^{N}
\end{array}, \text { for each } i=1, \cdots, N\right.
$$

The emotion detection algorithm returns the emotion $\zeta_{i}$ that represents a set of words $\psi=\left\{e_{1}, e_{2}, \cdots, e_{l}\right\}$. The algorithm uses Equation (6) to compute the model value for each emotion for the example $\psi$. Therefore, it calculates the highest value and then returns the index of this value. This index is used to determine the emotion. 


\section{Experiments and Results}

This section presents two purposes. First purpose is used to build Emotion Dominant Meaning Tree. The second purpose is to test the accuracy of using this tree for detecting the emotion.

\subsection{Data Sets}

The dataset uses ISEAR dataset [9] that contains emotional statements. ISEAR contains 7666 sentences (as shown in Table 1). The dataset is collected from 1096 participants with different cultural background who completed questionnaires about seven emotions: anger, disgust, fear, sadness, shame, joy, and guilt.

Emotion Detection Algorithm $\left(\psi=\left\{e_{1}, e_{2}, \cdots e_{l}\right\}, E=\left\{\zeta_{k}\right\}_{i=1}^{7}\right)$

- For $k=1$ to 7

○ Compute $\Omega_{k}(\psi)$

- $\operatorname{High}=0$; index $=1$;

- For $i=1$ to 7

$\circ$ if $\left(\Omega_{i}(\psi)>\right.$ High $)$

○ $\operatorname{High}=\Omega_{i}(\psi)$, index $=i$

- Return $\zeta_{i}$

\subsection{Building Emotion Dominant Meaning Tree}

Most of text classification methods use keyword-based methods with thesaurus. In contrast, we use the dominant meaning methods as features to improve accuracy and refine the categories. To build the dominant meaning tree, we use $60 \%$ of ISEAR dataset for seven emotion categories (as shown in Table 2): anger,

Table 1. Characteristics of the ISEAR Dataset.

\begin{tabular}{cc}
\hline Emotion & No. of Examples \\
\hline Anger & 1096 \\
Disgust & 1096 \\
Fear & 1095 \\
Sadness & 1096 \\
Shame & 1096 \\
Joy & 1094 \\
Guilt & 1093 \\
Total examples & 7666 \\
\hline
\end{tabular}

Table 2. Characteristics of dataset used to build tree.

\begin{tabular}{cc}
\hline Emotion & No. of Examples \\
\hline Anger & 658 \\
Disgust & 658 \\
Fear & 657 \\
Sadness & 658 \\
Shame & 658 \\
Joy & 656 \\
Guilt & 656 \\
Total examples & 4601 \\
\hline
\end{tabular}


disgust, fear, sadness, shame, joy, and guilt.

Stop words were removed in all examples for examples: for, an, the, a, an, another, but, or, yet, so, towards, before, etc.

Based on the Equation (1) to (5), we can build the dominant meaning tree of seven emotion categories, as shown in Figure 2.

Each node contains one emotion. Each emotion is associated with top- $N$ dominant meaning words based. The node between word and the emotion is labeled with its dominant meaning probability as shown in Figure 2. To determine $N$ value, we have to conduct some experimentations with different $N$ values to figure out which $N$ reflects a considerable results. The following subsection presents the accuracy of the proposed method to classify emotion examples.

\subsection{Detecting Algorithm Accuracy}

The goal of the experiments is to measure the accuracy of the proposed algorithm to predict a single emotional label given an input sentence. We follow Cohen's Kappa [10] to measure the accuracies of the experiment. We use average precision, recall, and F-measure to measure the classification accuracy.

In this experiment, we use ISEAR dataset to figure out the performance of our proposed mechanism. We used a Java programing language to create a class file to implement Emotion Detection Algorithm. This program classified the tested data in one emotion. The results of precision and recall are shown in Figure 3.

The precision and recall of our proposed approach shows a considerable performance comparing to those in related works.

In his classification he found that using SVM produced better results for sadness $(F 1=0.733)$ which is better than our approach for sadness $(F 1=0.67)$. In contrast, our approach has better results in others classes such as anger $(F 1=$ $0.66)$, disgust $(F 1=0.47)$, fear $(F 1=0.56)$, shame $(F 1=0.55)$, joy $(F 1=0.58)$, and guilt $(F 1=0.50)$. Where Balahur results were for anger $(F 1=0.38)$, disgust

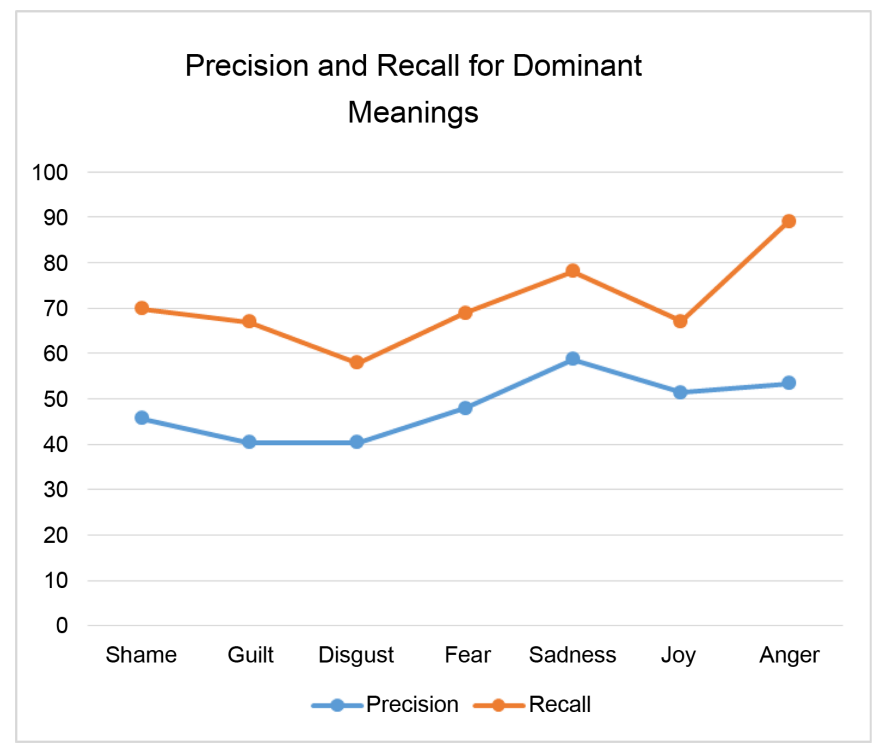

Figure 3. Precision and recall for dominant meanings. 
$(F 1=0.264)$, fear $(F 1=0.49)$, shame $(F 1=0.43)$, joy $(F 1=0.46)$, and guilt $(F 1=$ $0.42)$.

Danisman and Alpkocak [11] used the ISEAR collection and used vector space models (VSM) to categorize 801 examples. Our approach showed a significant results anger $(F 1=0.38)$, joy $(F 1=0.46)$, and sadness $(F 1=0.67)$ compared to Danisman and Alpkocak (2008) for anger $(F 1=0.242)$, joy $(F 1=0.496)$ and sadness $(F 1=0.371)$.

On the other hand, in order to test the performance of our proposed approach with alternative methods for emotion detection, we chose the work done by Balahur et al. [12], as shown in Table 3.

The results of 10-fold cross validation using Support vector machine to classify the whole set of 1081 examples initially chosen. We found that using dominant meaning classifier produced better results all categories than using the method of SVM in Balahur et al. [12], as shown in Table 3, where our proposed method produced the most accurate results for Sadness class with Precision (27.2) and Recall (60.2). Using 10-fold cross validation with SVM (Balahur, 2011) produced also the most accurate results for sadness class with Precision (0.707) and Recall (0.77). However, our proposed method produces a lower value for precision for two classes "Anger" with (20.2) and "Shame" with (20.2), Balahur's results produced a lower value for precision a class "Disgust" with 0.292 .

Figure 4 shows $F 1$ measure for the results of Dominant meaning approach and 10-fold cross validation using SVM [12]. As we see both our proposed approach and Balahur's approach have a similar function for drawing $F 1$ measure. We see that the top value for the graph for both approach recorded for "Sadness" class and the bottom value for the graph for both approach recorded for "Disgust" class.

\section{Conclusion}

Text-Based Emotion detection becomes an important research field with the massive chatting messages coming from social media systems. In this paper, we have proposed an approach to extract user's emotion based on messages who posts. We used a dominant meaning approach, which looks for the meaning of the word rather than the word itself. To do that, we proposed an architecture for

Table 3. Characteristics of dataset used to build tree.

\begin{tabular}{ccccc}
\hline \multirow{2}{*}{ Emotion } & \multicolumn{2}{c}{ Precision } & \multicolumn{2}{c}{ Recall } \\
\cline { 2 - 5 } & Our method & Balahur & Our method & Balahur \\
\hline Anger & 20.2 & 0.353 & 52.1 & 0.414 \\
Disgust & 22.4 & 0.292 & 46.9 & 0.241 \\
Fear & 26.2 & 0.482 & 55.7 & 0.491 \\
Guilt & 20.3 & 0.462 & 51.9 & 0.386 \\
Joy & 26.6 & 0.439 & 50.6 & 0.474 \\
Sadness & 27.2 & 0.707 & 60.2 & 0.76 \\
Shame & 20.2 & 0.441 & 48.9 & 0.412 \\
\hline
\end{tabular}




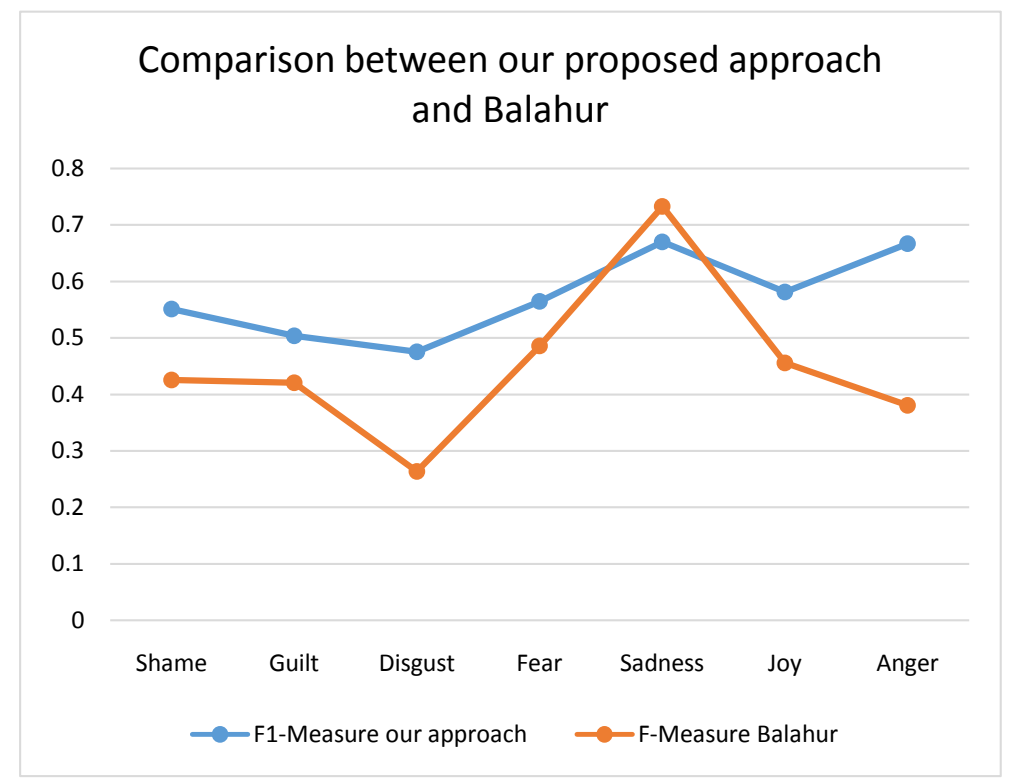

Figure 4. Comparison between the results of dominant meaning approach and 10-fold cross validation-using SVM (Balahur, 2011).

the proposed system to finish two tasks: training and classification. For training system, a hierarchy tree for dominant meaning for seven emotions ("joy, fear, anger, sadness, disgust, shame, and guilt") is built using ISEAR dataset. We create an algorithm called Emotion Detection Algorithm to classify and find the suitable emotion class based on the text. To experiment the proposed technique, we tested it on the ISEAR dataset, and compare our results with different results that were implemented by Alexandra Balahur [12] and Danisman and Alpkocak [11]. We show that our system has the best results in precision, recall and $F$-measure.

\section{References}

[1] Alm, C.O., Roth, D. and Sproat, R. (2005) Emotions from Text: Machine Learning for Text-based Emotion Prediction. Proceedings of the Human Language Technology Conference and the 2005 Conference on Empirical Methods in Natural Language Processing, Vancouver, Canada, 6-8 October 2005, 579-586. https://doi.org/10.3115/1220575.1220648

[2] Alm, C.O. (2009) Affect in Text and Speech. VDM Verlag, Saarbrücken.

[3] Chen, Y., Lee, S.Y.M. and Huang, C.-R. (2009) A Cognitive-Based Annotation System for Emotion Computing. Proceedings of the Third Linguistic Annotation Workshop (The LAW III), Suntec, Singapore, 6-7 August 2009, 1-9.

[4] Tokuhisa, R., Inui, K. and Matsumoto, Y. (2008) Emotion Classification Using Massive Examples Extracted from the Web. Proceedings of COLING, Manchester, 18-22 August, 2008, 881-888. https://doi.org/10.3115/1599081.1599192

[5] Jraidi, I., Chalfoun, J. and Frasson, C. (2012) Implicit Strategies for Intelligent Tutoring Systems. Cerri, S.A., et al., Eds., Springer-Verlag, Berlin Heidelberg, 1-10.

[6] Razek, M.A., Frasson, C. and Kaltenbach, M. (2003) A Context-Based Information Agent for Supporting Intelligent Distance Learning Environments. The 12th International World Wide Web Conference ( $W W W$ 2003), Budapest, Hungary, 20-24 
May 2003, 183-191.

[7] Wu, B. (2013) Appraisal Perspective on Attitudinal Analysis of Public Service Advertising Discourse. Published by Canadian Center of Science and Education, English Language and Literature Studies, Vol. 3, No. 1.

[8] Martin, J. (2003) Introduction, Special issue on Appraisal. Text, 23, 171-181.

[9] Scherer, K.R. and Wallbotth, G. (1994) Evidence for Universality and Cultural Variation of Differential Emotion Response Patterning. Journal of Personality and Social Psychology, 66, 310-328. https://doi.org/10.1037/0022-3514.66.2.310

[10] Cohen, J. (1960) A Coefficient of Agreement for Nominal Scales. Educational and Psychological Measurement, 20, 37-46. https://doi.org/10.1177/001316446002000104

[11] Danisman, T. and Alpkocak, A. (2008) Feeler: Emotion Classification of Text Using Vector Space Model. Proceedings of the AISB 2008 Symposium on Affective Language in Human and Machine, University of Aberdeen, UK, 1-4 April 2008, 53-59.

[12] Balahur, A., Hermida, J.M. and Montoyo, A. (2011) Building and Exploiting Emotinet, a Knowledge Base for Emotion Detection Based on the Appraisal Theory Model. IEEE Transactions on Affective Computing, 3, 88-101.

Submit or recommend next manuscript to SCIRP and we will provide best service for you:

Accepting pre-submission inquiries through Email, Facebook, LinkedIn, Twitter, etc. A wide selection of journals (inclusive of 9 subjects, more than 200 journals)

Providing 24-hour high-quality service

User-friendly online submission system

Fair and swift peer-review system

Efficient typesetting and proofreading procedure

Display of the result of downloads and visits, as well as the number of cited articles

Maximum dissemination of your research work

Submit your manuscript at: http://papersubmission.scirp.org/

Or contact jilsa@scirp.org 